\title{
Modeling perisaccadic time perception
}

\author{
Andrea Guazzini ${ }^{1}$, Pietro Liò ${ }^{2}$, Andrea Passarella ${ }^{1}$, Marco Conti ${ }^{1}$ \\ ${ }^{1}$ Institute of Informatics and Telematics-IIT-CNR, Pisa, Italy; \\ ${ }^{2}$ Computer Laboratory, University of Cambridge, Cambridge, UK \\ Email: andrea.guazzini@gmail.com
}

Received 29 July 2010; revised 15 August 2010; accepted 18 August 2010.

\begin{abstract}
There is an impressive scarcity of quantitative models of the clock patterns in the brain. We propose a mesoscopic approach, i.e. neither a description at single neuron level, nor at systemic level/too coarse granularity, of the time perception at the time of the saccade. This model uses functional pathway knowledge and is inspired by, and integrates, recent findings in both psychophysics and neurophysiology. Perceived time delays in the perisaccadic window are shown numerically consistent with recent experimental measures. Our model provides explanation for several experimental outcomes on saccades, estimates population variance of the error in time perception and represent a meaningful example for bridging psychophysics and neurophysiology. Finally we found that the insights into information processing during saccadic events lead to considerations on engineering exploitation of the underlying phenomena.
\end{abstract}

Keywords: Brain Clock; Saccades; Time Compression; Time Perception; Craniotopiccoordinates

\section{INTRODUCTION}

One of the most important research areas in neuroscience focuses on the neural mechanisms of time perception and in particular on the link between visual perception and the brain $\operatorname{clock}(\mathrm{s})$; for sake of simplicity, we will call this visual timing. In the last decade important evidences of a strong distortion of the time perception during saccadic eye movements have been attracted the most the attention of researchers. Saccades represents probably the perfect process to study "visual timing" both for the changing of external space mapping onto retina and for the brain pathways alterations caused by the saccadic neural correlates [1-6]. The numerical simulation of the known saccadic effects by a model which integrates the insights coming from psychophysics and neurophysiology appear today to be a promising scenario to extend the discussion on the brain $\operatorname{clock}(\mathrm{s})$ problem. One interesting question is whether temporal judgments is distributed or centralized [7]. The time perception in the range of milliseconds to minutes involves variety of neural population located in the frontal cortex, hippocampus, basal ganglia and cerebellum. Nowadays it is well known that the "internal" representation of time depends on the integration of multiple neural systems. While there is good evidence about different clocks for different interval lengths, some recent evidences point clearly to the existence of visually based timing mechanisms. Researchers from psychophysics and neurophysiology have approached this topic from different sides, both leading to experimental evidences that can be now merged together to obtain a more complete perspective for visual timing processes than in the past. The description of the neurological structures can be performed at different scales. At the lower level of description, the micro-scale is represented by single neurons as nodes. Using a complex network framework, we can assign certain properties of individual nodes (degree, centrality, clustering, etc.); in order to model the dynamical process each node is performing in processing the signal. This level of description is unfortunately computationally very expensive nowadays and very precise only for small networks; therefore it does not allow a generic analysis of the global properties of the system. At the other extreme, we have the higher level of description, the macro-scale, represented by the psychophysics where statistical properties of the network as a whole. In the middle of these descriptions still remains a huge space for different scales of descriptions that are termed mesoscales, or intermediate scales. These scales are understood as substructures (eventually sub graphs) that have topological entity compared to the whole network, e.g. populations/communities of neurons which are functionally and/or structurally homogeneous. In particular, the community detection problem concerning the determination of mesoscopic structures that have functional, relational or even social entity is still contro- 
versial, starting from the a priori definition of what a community is. The correct determination of the mesoscale in complex networks is a major challenge. On the mesoscopic level, researchers have examined neural activity as measured by means of local field potentials or dense intracranial electrode arrays in human and non-human subjects. These approaches have stimulated models of neuronal communication between nearby regions. By contrast, macroscopic parameters of neural activity have been used to assess the characteristics of large neuronal assemblies that fire in synchrony and thus generate detectable voltage or magnetic gradients on the surface of the scalp. This paper originates from our participation in the three years EU project MEMORY in which large amount of data has been collected. On the light of the MEMORY project and discussions with experimentalist we aim at devicing the model that could answer the question of time perception during the saccadic events.

\subsection{Combining Neurophysiological and Psychophysical Evidencies}

The psychophysical studies of the relationship between a visual stimulus (say a dot moving etc) and the perception of the elapsed time between the start and end of the stimulus (which is subjective) have produced important results. The finding that perceived duration of a dynamic stimulus can be manipulated in a local region of visual space has confirmed that the visual perception of time is processed directly by superior visual areas at least in its early stages [8]. An interesting experiment has recently shown that the existence of a large contrast effects in the discrimination of short temporal intervals during fixation events [9]. Della Rocca et al. examined the effect of temporal distractors on interval discrimination and showed that the perception of the time driven by visual events is affected by early past experiences, as it happens for others characteristics related to the visual stimuli such as the shape, color or movement of objects. An important finding comes from the studies of Morrone and Burr about time perception during saccades [4]. Their major results has been that perceived time is compressed when stimuli are ashed shortly before or after the onset of a saccadic eye movement $[1,2,10]$. Moreover time and space perception seem to follow the same compression pattern during saccades both for timescale and magnitude of the effects. This evidence suggests that time information is embedded with other characteristics in the same neural representation of stimuli. The neurophysiological approach to the visual timing has revealed neural correlates of visual experience which could account for the psychophysical findings [11-13]. The hypothesis of an explicit neural representation of temporal attributes of visual stimuli has a wide acceptance [14]. From this point of view the fundamental representation of visual information should be able to encode temporal information similarly to the other characteristics of the perceived matter (color, frequency, brightness). Previous studies have drawn the hypothesis that cerebral circuits are inherently able to rescale durations in a proportional manner and compensate for the error differences generated by the cerebellum. Following such assumption, to explain visual timing failures during saccades an explicit model has to consider at least those neural areas and pathways by which visual experience is represented, filtered and analyzed [15]. In a recent paper, Buonomano proposed that short-term plasticity and dynamic changes in the balance of excitatory-inhibitory interactions may underlie the decoding of temporal information, that is, the generation of temporally selective neurons [16]. He first showed that it is possible to tune cells to respond selectively to different intervals by changing the synaptic weights of different synapses in parallel. Short-term plasticity is a usage-dependent change in synaptic strength on the time scale of millisecond to seconds and is observed in almost every synapse types of the central nervous system. Each type of synapse has its own specificity with respect to this property. When stimulated a few times within a second, some synapses show facilitation, others depression or else complex sequences of facilitatory and depressing changes [16]. According with the previous claim, recent results show that for visual perception the timing attributes of visual experience and the temporal tuning properties of certain visual neurons are linked. Moreover neurons in visual areas of primate parietal cortex have reduced latencies to visual stimulation at the time of a saccade [3]. Several hypotheses of time measuring systems, peculiar to neural circuits, demonstrates synchronization enables both the evaluation of different time scales and the binding between different sensorial/cognitive modalities. Lesion and functional imaging studies have identified frontal, temporal, and parietal areas as playing a major role in the control of visual timing processing, but very little is known about these areas interact to form a dynamic attentional network. We know that the frontoparieto-temporal (FPT) network communicates by means of neural phase synchronization. Results reveal that communication within FPT proceeds via transient longrange phase synchronization in the beta bend [17]. Recent results concern the high-frequency long range coupling between prefrontal and visual cortex. Desimone et al. used paired recordings in the frontal eye field (FEF) and area V4, they found that attention to a stimulus in their joint receptive fields leads to enhanced oscillatory cou- 
pling between the two areas, particularly at gamma frequencies [18]. This coupling appeared to be initiated by FEF and was time-shifted by about 8 to $10 \mathrm{~ms}$ across a range of frequencies. Considering the expected conduction and synaptic delays between the areas, this time-shifted coupling at gamma frequencies may affect the postsynaptic impact of spikes from one area upon the other, consequently affecting visual timing [18]. This long-range synchronization mechanism could be crucial especially during saccades, where we know those 100 ms before the saccade onset the FEF releases a corollary discharge signal towards the lateral intraparietal cortex LIP, where a craniotopical representation of the environment is implemented. The receptive fields of visual neurons are known to be retinotopically arranged, and in awake animals they move with gaze, maintaining the same retinotopic location regardless of eye position. Nevertheless the existence in the monkey parietal cortex of cells (called "real-position" cells) whose receptive field does not systematically move with gaze has been proven [19]. These cells respond to the visual stimulation of the same spatial location regardless of eye position and therefore directly encode visual space in craniotopic instead of retinotopic coordinates [20]. The desynchronization due to the long-range coupling between FEF and V4 tell us that the remapping process act initially on the older visual signal, while the present one will be encoded later. Finally at least two considerations have to be taken into account in order to elucidate the neurophysiological implications on the visual timing during saccades. As shown in Figure 1, the former is a delay between the stimulus administration and its encoding into the craniotopical representation of the world allocated in LIP, and the latter is that this delay is increased at the time of saccades because of the different neural networks involved in the fixation and the saccadic state.

\subsection{Communication Network Approach}

In modern neuroscience, computational methods occupy an important niche. Neuroscience computing is concerned with building mathematical and statistical models to address a wide range of questions originated from experimental neurobiology and related medical fields. Typically, these models involve massive, hardware-demanding amounts of calculations performed on last-generation computers. However, the complexity of real-life biological systems is indeed overwhelming, far outreaching the abilities of modern computing systems. This creates a considerable demand for parsimonious models which capture main driving forces of biological processes elucidating the mechanisms behind the observed phenomena. The search for a model which is

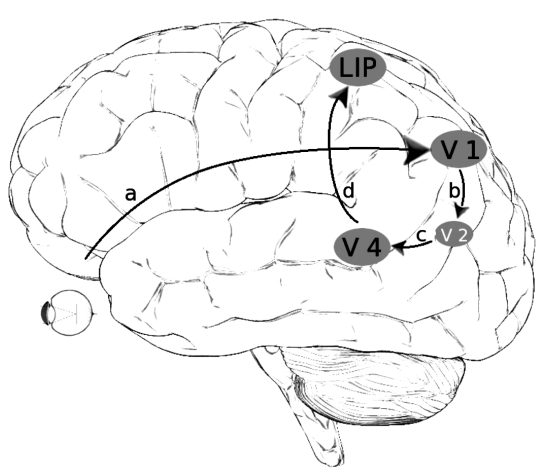

(a)

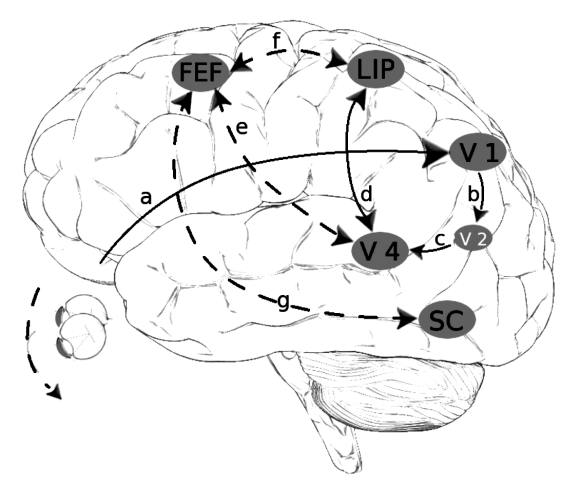

(b)

Figure 1. Schematic representations of the neural circuits of early stage of vision process, respectively involved in the fixation condition (a-simplified neural network of vision during fixation) and during a saccadic onset (b-neural network during saccades). From a to $g$ are indicated the links among the vertex which form the pathway.

complex enough to be a reasonable approximation of the biological reality and at the same time simple enough to be manageable by available mathematical techniques is some sort of an art. The task requires the researcher not only to have a thorough understanding of the relevant biology and mathematics but sometimes a great deal of luck to have the predictions of the model to be consistent with the natural phenomena. Computational neuroscience focuses on two distinct levels of description: 'simple models' and 'detailed models'. While detailed models focus on the measurement and description of complex systems through using certain building blocks, their interactions and dynamic properties, such as kinetic parameters, binding constants etc. Simple models aim at capturing the behavior of the few most relevant components of the system. This implies that such models are also intrinsically more abstract than detailed models their components and parameters often do not correspond directly to well-defined physical quantities, such as measured binding constants or chemical reaction rates. 
Nonetheless, simple models can provide insights into the behavior of a system and drive experimental and detailed modeling efforts by suggesting further more detailed experiments or models. Moreover such modeling framework could allow easier predictive multi-scale descriptions of the system under study than detailed models. Current detailed models are based on two different approaches: a bottom-up approach which focuses on the measurement and description of complex systems using the building blocks their interactions and dynamic properties, a top-down modeling approach attempts to develop integrative and predictive multi-scale models of the system under study. Our goal is to develop a hybrid strategy between the two frameworks, focusing on specific problems at particular scales using a simple modeling and then expand on other scales. A main target of using such combination of modeling approaches is to get deeper understanding of the system without suffering from sudden growth of degrees of freedom. A coherent approach to study visual timing should take into account the neurophysiological knowledge about the fronto-parieto-temporal network, providing for first a simplified model of its functional structures. Afterwards throughout a reverse engineering process would be possible to validate the model fitting the psychophysical data. Such approach can provides insights about the way by which the brain has optimized its "software" to measure the time in the absence of clock, with respect to its own biological constraints. In order to represent the complexity of the visual timing processing, it seems to be essential to define at least three computational objects. The first object is an appropriate structure of the neural representation for the visual information. From psychophysics we know mainly that visual information preserves a craniotopic structure from early to late stages of brain analysis. From these evidences the system seems to be characterizable by coupling neural pathways, where the neural signal coming from visual ganglion cells propagates. Moreover real neural structures are able to embed spatial and temporal information about visual stimuli in the same neural representation, and by a phase of synchronization dynamics among FPT, are able to represent directly the time information. The second aspect that is necessary to manage relies on the signal propagation dynamics along the structures defined above. As shown by recent experiments the dynamics of signal propagation could have a role in the visual timing, and to reproduce their peculiarity both the synchronization of the neurons' activity and the dynamics of synapses have to be taken into account. An appropriate representation of the synaptic learning parameters is to incorporate in the model the insights of Buonomano about the relation between synapses short term dynamics and the capacity of neural structure to represent the timing aspects of a perceived stimulus. The last behavior which has to be captured is the phase synchronization dynamics which characterized the FPT network. To face this challenge a phase dynamics of the neural signal is explicitly modeled, and a correlation between this parameter and signal propagation is shaped by the equations of the model.

Finally the main attempt of this paper will be to highlight the interplay between the signal diffusion and the information diffusion. The paper proceeds as follows, in the Section 2 the computational model is presented and their component is elucidated. Section 3 describes the numerical simulations of the psychophysical experiments taken into account, and in Section 4 the results are presented. Finally in the Section 5 numerical results and insights are discussed and next steps and conclusion are outlined.

\section{THE MODEL}

\subsection{The FEF-LIP-V4 Network}

The cerebral network FEF-LIP-V4 is represented by a cellular automata composed by interconnected elements labeled as nodes, where the retinotopic input propagates and that allocate the spatiotopic layer. The cellular automata can be seen as having one 'space dimension' which are nodes describing clustered or homogeneous populations (e.g. column), and one orthogonal 'time dimension' which encode the duration of the stimuli and the interval among them (Figure 2). Our aim is to investigate the visual timing related aspects without introducing particular topology. As a consequence we image to have a neurons lattice where the distances among nodes are related to the transfer of the stimulus information, from one node ito the next one $\mathrm{i}+1$, and are assumed to be equivalent. The purpose of this equivalence of time transfer is to avoid explaining time space aberration through the introduction of a bias or fictitious, ad hoc mechanisms. Cerebral pathways connecting V4 with LIP and FEF are approximated by parallel neural delay chains

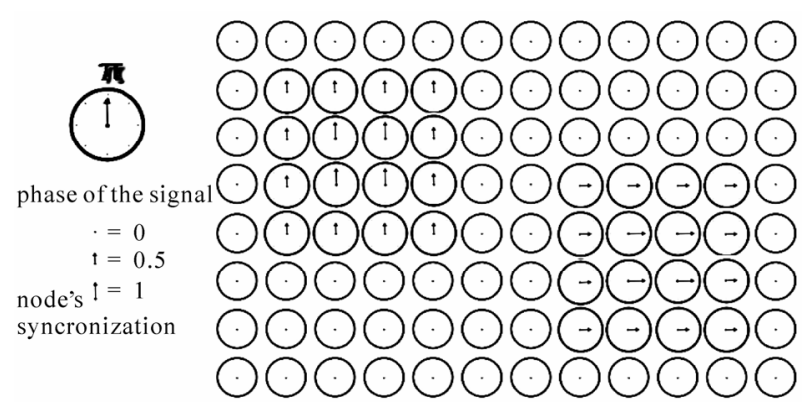

Figure 2. Schema of two signals moving from left to right, which are identified by their phases (arrow) and its intensity (arrow's length). See legend on the left. 
of nodes, where the signal propagates synchronizing subsequent nodes' phase within a column, and modifying the weight of synapses among nodes. The cellular automata can be seen as having one 'space dimension' which are nodes describing clustered or homogeneous populations (e.g. column), and one orthogonal 'time dimension' which encode the duration of the stimuli and the interval among them.

Each node represents a homogeneous population of neurons; nodes are spatially organized in parallel columns to reproduce the pathway followed by the neural signal coming from visual area V4. Neurons inside a node are assumed to synchronize their activity whenever a signal arrives, e.g. to fire simultaneously, and are linked together by synapses with a long term dynamics [21]. The node is assumed as the "atomic" component of the system and its behavior is explicitly modeled by the definition of an activation state $S_{i, j}{ }^{t}$ within the interval $(0$, 1). Given the physiological constraints three different contributions to the signal have to be taken into account. Besides the contribution of the direct synapses it is needed to consider the lateral connections (transcolumnar contribution) and the respective signals' phases in order to calculate the resulting signal which is summed to the target node. The synaptic weights inside a column are indicated as $\left(W d_{i, j}{ }^{t}\right)$ while the transcolumnar synaptic weights are indicated as $\left(W l_{i, j}{ }^{t}\right)$.Using the trigonometric formulas one can write the contributions as follows:

$$
\begin{gathered}
I_{T}=\left(S_{i-1, j-1} W l_{i-1, j-1}\right)^{2}+S_{i-1, j} \frac{\tanh \left(\beta_{1}\left(W d_{i, j-1}-\beta_{2}\right)\right)+1}{2}+\left(S_{i+1, j-1} W l_{i+1, j-1}\right)^{2} \\
I_{1}=2\left(S_{i-1, j-1} W l_{i-1, j-1}\right)\left(S_{i, j-1} W d_{i, j-1}^{2}\right) \cos \left(\phi_{i-1, j-1}-\phi_{i, j-1}\right) \\
I_{2}=2\left(S_{i-1, j-1} W l_{i-1, j-1}\right)\left(S_{i+1, j-1} W d_{i+1, j-1}^{2}\right) \cos \left(\phi_{i-1, j-1}-\phi_{i+1, j-1}\right) \\
I_{3}=2\left(S_{i, j-1} W d_{i, j-1}^{2}\right)\left(S_{i+1, j-1} W l_{i+1, j-1}\right) \cos \left(\phi_{i, j-1}-\phi_{i+1, j-1}\right)
\end{gathered}
$$

The incoming signals are consequently computed using the Eq.5 and the activation state of each node are finally updated according with the Eq.6.

$$
\begin{gathered}
\hat{S}_{i, j}^{t+1}=\sqrt{I_{T}+I_{1}+I_{2}+I_{3}} \\
S_{i, j}^{t+1}=S_{i, j}^{t}+\hat{S}_{i, j}^{t+1}
\end{gathered}
$$

In order to introduce the long range synchronization effects within the V4-LIP-FEF network the phase of each node is explicitly considered assigning to each of

them a value $\varphi_{\mathrm{ij}}{ }^{\mathrm{t}}$ within $(-\pi,+\pi)$. Therefore the incoming signals have to be described as having their own magnitude and phase. To estimate the resulting phase we use again the trigonometric formulas:

$$
\begin{gathered}
S_{1}^{t}=S_{i-1, j-1}^{t} W l_{i-1, j-1}^{t} \\
S_{2}^{t}=S_{i, j-1}^{t} W d_{i, j-1}^{t} \\
S_{3}^{t}=S_{i+1, j-1}^{t} W l_{i+1, j-1}^{t}
\end{gathered}
$$

Then the phase dynamics of a node has been coupled with the magnitude of the signal coming from the connected nodes and the state of the node itself, as follows:

$$
\varphi_{i, j}^{t+1}=\arctan \left(\frac{\hat{S}_{i, j}^{t} \sin \left(\hat{\varphi}_{i, j}^{t}\right)+S_{i, j}^{t} \sin \left(\hat{\varphi}_{i, j}^{t}\right)}{\hat{S}_{i, j}^{t} \cos \left(\hat{\varphi}_{i, j}^{t}\right)+S_{i, j}^{t} \cos \left(\hat{\varphi}_{i, j}^{t}\right)}\right)
$$

A way for the brain to encode in the same neural signal both time and space information about a stimulus, could be to exploit the network V4-LIP as a sort of neural buffer. Assuming linked nodes which evolve "rapidly" their connections weight according with the incoming signal features (e.g. activation and phase agreement), it is possible to equip an artificial system with a sort of embedded short memory of past signals or in another words with a state dependent response behavior to the signal. Such hypothesis has led Buonomano to explore the role of synapses characterized by a short term dynamics [16], demonstrating how a neural network equipped with such synapses can represent both spacial and temporal information about the stimulus by the same neural correlates on a spatiotopic map. In order to take into account the role of the synapses' dynamics on both the signal dynamics and the node's synchronization the equation for the synaptic enforcement has been shaped including this features, according with the biological constraints. The synaptic weight $\left(W_{i, j}{ }^{t}\right)$ is defined as a variable of the model belonging to the interval $(0,1)$. 
To design the complex dynamics of the synapses two ad-hoc parameters are designed and labeled respectively learning factor $(\gamma)$ and decay factor $(\delta)$, both defined within $(0,1)$. Since the physiological characteristics of the ocular dominance columns we introduced two different values to distinguish between the signals' dynamics inside and outside the column. Consequently we indicate the intracolumnar and the transcolumnar learning and decay factor respectively as $\gamma_{d}$ and $\delta_{d}$ for the intracolumnar synapses and $\gamma_{l}$ and $\delta_{l}$ for the transcolumnar (e.g lateral) synapses. Moreover two thresholds constants are introduced to indicate the resting values for the synaptic weight $\left(K_{w}\right)$ and the nodes' activity $\left(K_{s}\right)$, both within the interval $(0,1)$. The synaptic weight is finally updated at each time step using the following recipe. If the activity of the precedent node is greater than the activity resting threshold $\left(K_{s}\right)$, according to Hebb's theory [22], the synaptic weight increases with the signal magnitude and the phase synchronization between the two connected nodes. Otherwise the synapses would decay proportionally with the distance between phases of connected nodes and as a function of their activity (Eqs.13, 14).

$$
\begin{gathered}
\Delta \phi_{i, j: i, j+1}^{t+1}=\frac{\min \left(\left|\varphi_{i, j}^{t}-\varphi_{i, j+1}^{t}\right|\right)}{\pi} \\
W d_{i, j}^{t+1}=W d_{i, j}^{t}+\gamma_{d}\left(1-\Delta \phi_{i, j: i, j+1}^{t+1}\right)^{2}\left(S_{i, j}^{t}-K_{s}\right) \\
-\delta_{d} \Delta \phi_{i, j: i, j+1}^{t+1}\left(W d_{i, j}^{t}-K_{w}\right)\left(1-\left(W d_{i, j}^{t}-K_{w}\right)\right) \\
W l_{i, j}^{t+1}=W l_{i, j}^{t}+\gamma_{l}\left(1-\Delta \phi_{i, j: i \pm 1, j+1}^{t+1}\right)^{2}\left(S_{i, j}^{t}-K_{s}\right) \\
-\delta_{l} \Delta \phi_{i, j: i \pm 1, j+1}^{t+1}\left(W l_{i, j}^{t}-K_{w}\right)\left(1-\left(W l_{i, j}^{t}-K_{w}\right)\right)
\end{gathered}
$$

Finally the main assumption relies on the different learning and decay functions for intracolumnar and extracolumnar synapses dynamics. In the Eqs.13 and $\mathbf{1 4}$ the parameters $\gamma_{d}$ and $\gamma_{1}$ rule the learning rate respectively for intracolumnar and transcolumnar synapses.

\subsection{Numerical Simulations}

In order to study the phenomenology of the computational model we started grounding on few basic assumptions the design of the control parameters under consideration. The decay dynamics is controlled by the parameters $\delta_{\mathrm{d}}$ and $\delta_{\mathrm{l}}$. The different nature of intracolumnar and transcolumnar connections is expressed also in terms of signal propagation effciency. While the slow dynamics of the transcolumnar synapses is coupled with a linear function of signal magnitude transmission $[1,20,23]$, the faster dynamics of the intracolumnar synapses has been shaped by a sigmoidal behavior [24,25]. Another fundamental assumption relies on the spatiotemporal transformation at the neural level, here proposed as the fundamental key-mechanism for the visual time perception. Our approach is based on a fundamental symmetry between the neural buffer and the phases based theories for the neural activities. Whether we assume the neural propagation of the signal as a phase dynamics of different neuronal populations the equations get biological sense. Consequently the more computationally light approach has been adopted, implementing the time related phase dynamics of a node as the degree of diffusion of the signal along a neural delay chain. The retinotopic input of a real stimulus is simulated by the imposition of a pick of activation in those nodes of the first layer of the automata, corresponding to the spatiotopic representation of the stimulus. The activation of the local populations of nodes is maintained for the entire duration of the encoded stimulus. The nodes which encode the stimulus are assumed as synchronized and characterized by the same phase for the entire duration of the stimulus. At the end of the stimulus the synchronization inside and within the nodes decreases following the Eq.11. Among the peculiarities of the saccades, there is the recalibration of the spatiotopicityin the encoding stage of visual processing (LIP) which anticipate them. This recalibration is reached by the FEF-LIP-V4 network by mean of an internal signal representing the eye position defined corollary discharge [26]. The corollary discharge (CD) starts $60 \mathrm{~ms}$ before the saccade from the FEF and is complete at saccadic offset producing an apparent enlargement of the visual receptive fields, and a fast remapping of the visual environment, biasing both time and space detection ability. In order to simulate the CD effects on the craniotopical representation of the visual stimuli, in the remapping phase simply we shift the signal parallel to the direction of the saccade. In this way while a stimulus temporally distant from a saccade (i.e. long after or before) produces the same response in the craniotopic map, during or in the temporal proximity (50-100 ms) at the saccadic event the activation and synchronization pattern of the map appear as altered with respect to the fixation condition. As a consequence of this alteration in the read out stage the system could fail to estimate the time features of the stimuli. In humans the "readout" task has to operate on the craniotopic representation of the early visual history, in order to reveal both topological distances between stimuli and timing features as duration of an interval between two visual events. The model we have defined operates a temporal to spatial transformation of the neural signal, producing a representation of the craniotopic map allocated in (LIP). The state of this map has been analyzed by a two step procedure: for first the state of synchronization of the map were detected by a kohonen neural 
network [27], obtaining the absolute "network position" of their trace of synchronization and finally these positions were used by trained output neurons to estimate the temporal localization of the stimuli. The system appears to be able to detect veridically time and space localization of a stimulus in fixation conditions Figure 3.

During the saccadic event two main consequences could be postulated given our mesoscopicalmodelization of the system. As shown in a previous work [28] the former is determined by the remapping of the signal literally over previous activated pathway, at least in the LIP neural district. The latter is the delay between the peripheral detection of the signal and its encoding in the

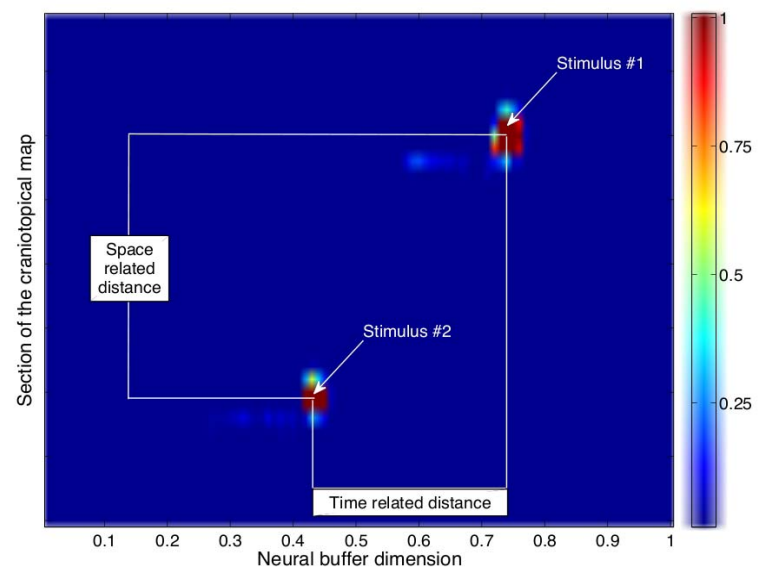

(a)

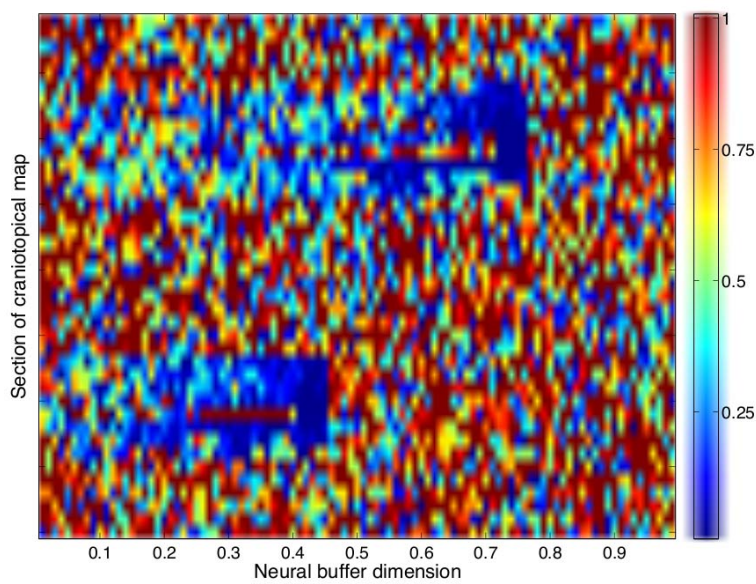

(b)

Figure 3. Figure a and $\mathrm{b}$ represent the magnitude of activation and the phases synchronization of the ensemble of nodes respectively. The craniotopical map is idealized by a region described in xycoordinates; in a we show the magnitude of nodes' activation, and in $b$ the phase difference with the first neighbors. The color bars on the right indicate a normalized intensity variation both for the activation and the phases differences.
LIP district due to the different pathways which are activated. To fit the model parameters a real psychophysical experiment about time compression during saccades has been taken into account and used as reference dataset. In the considered experiment Burr et al. studied the perception of time during saccades asking the subject to compare the time interval between two couples of consecutive visual stimuli in two different conditions, during fixation and during a saccadic event [2]. The experimental results have allowed us to analyze the relation between the temporal proximity of a saccadic event and the magnitude of time compression. To simulate the experimental paradigm we define two temporal variables labeled respectively as $T_{\text {sac }}$ for the interval between the first stimulus administration and the saccadic onset, and $\delta \mathrm{T}_{\mathrm{st}}$ to indicate the interval between the stimuli. The final stage of the numerical simulations has generalized the psychophysical paradigm described in Ross et al. [5] about psychophysical effects of time compression during saccades for different values of both the delay between the visual stimuli and their temporal distance from the saccade onset.

\section{RESULTS AND DISCUSSION}

Following the schema described in Figure 2 we simulate in Figure 3 the activation of nodes in a region of craniotopical map. Signals of different magnitude (a) are identified by the change of phase (b). The first task of the numerical simulations has been to validate the model using the experimental data gently provided from Morrone et al. [4]. After some preliminary simulations we found how the "transcolumnar" learning and decay factors only determine the lateral diffusion/synchronization of the signal, having no direct effects on the time estimation. As a consequence these parameters have been treated as nuisance variables and set to values of convenience. Finally the "intracolumnar" learning and decay factors have been estimated fitting the real data by a standard least square method. First we defined a reference dataset starting from the real data, which provided the time compression of two visual stimuli separated by $100 \mathrm{~ms}$ depending on the saccade's onset time. Then we compute the least square distances between the model estimations and reference dataset for different values of $\gamma_{\mathrm{d}}$ and $\delta_{\mathrm{d}}$ (Figure 4). The best fitting curve is shown in figure 6 and it is obtained for $\gamma_{d}=0.07$ and $\delta_{d}=0.08$ and characterized by an $r^{2}=0.93$. The space of parameters in Figure 4 shows those values for which the system is characterized by a coherent behavior with the real data. Finally these values have been assumed as parameters of the model for the second phase of numerical simulations.

The second task of numerical simulation has been the exploitation of the model in order to explore its behavior 


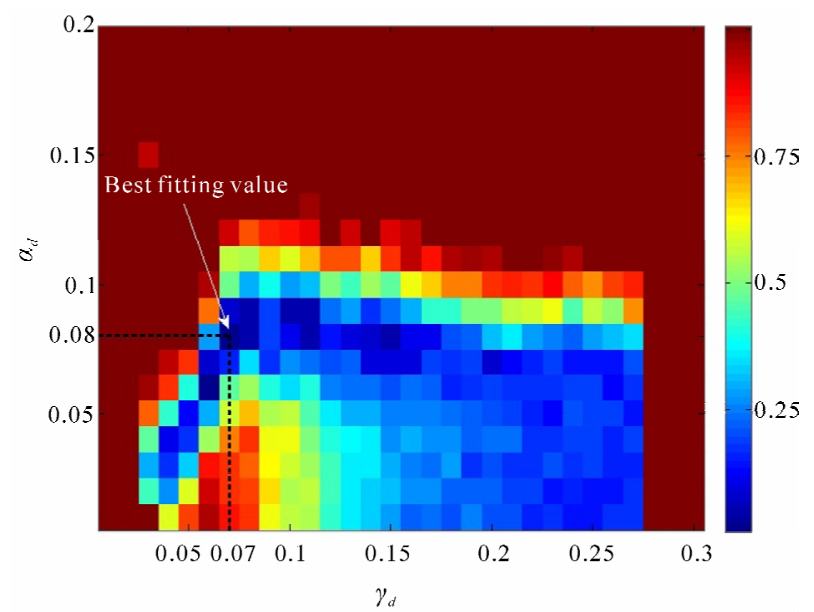

Figure 4. Space of parameters for $\gamma_{d}$ and $\delta_{d}$ respectively on the $\mathrm{X}$ and $\mathrm{Y}$ axes. The color (see legend) indicates the normalized least square distance between the model estimation and the real data. The best fitting value is obtained for $\gamma_{d}=0.07$ and $\delta_{d}=$ 0.08 .

for different values of delay between the visual stimuli. The estimation of the delay between two subsequent stimuli separated by an interval of $\Delta \mathrm{T}_{\text {st }}$ is considered both for different values of the saccadic onset $T_{\text {sac }}$, and for different values of $\Delta \mathrm{T}_{\mathrm{st}}$. The percentage of time compression is computed comparing the model's interval estimations in the fixation condition with those near the saccade, and it was assumed as order parameter.

The normalized time compression among the two conditions is reported using colors, with red for large values of compression and blue for compression near to 0 . The $y$ axis indicates the delay between the time of the saccade onset and the time of the first administered stimulus while the $\mathrm{x}$ axis indicates the delay between the couple of stimuli. Since the available experimental data about this paradigm are only estimated for a delay among the stimuli of $100 \mathrm{~ms}$ these results provide some predictions/forecasting which would be interesting to verify.

The Figure 5 shows how the maximum of compression is obtained always around the saccadic onset. Pairs of stimuli separated by a little delay appear as more compressed when administered largely before the saccadic onset, and exists a critical values of $200 \mathrm{~ms}$ for which no significant compression is revealed.

\section{CONCLUSION}

We have developed a model that provides a fit of experimental data concerning time compression during saccades. With this work we suggest a way to couple the craniotopical representation of space-time attributes of a visual stimulus with the neurophysiological structure of the brain. The model has been tested through using two parame-

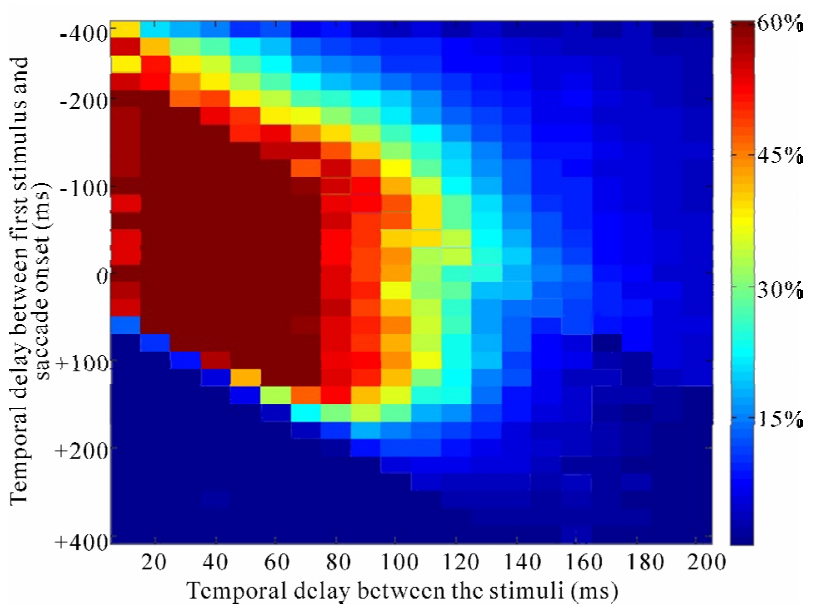

Figure 5. Time compression (see legend) is indicated as the normalized difference between the time estimation in the fixation condition and in the saccadic condition. In y axis we show the delay between saccade onset and the first administered stimulus. In $\mathrm{x}$ axis the delay between the stimuli is represented.

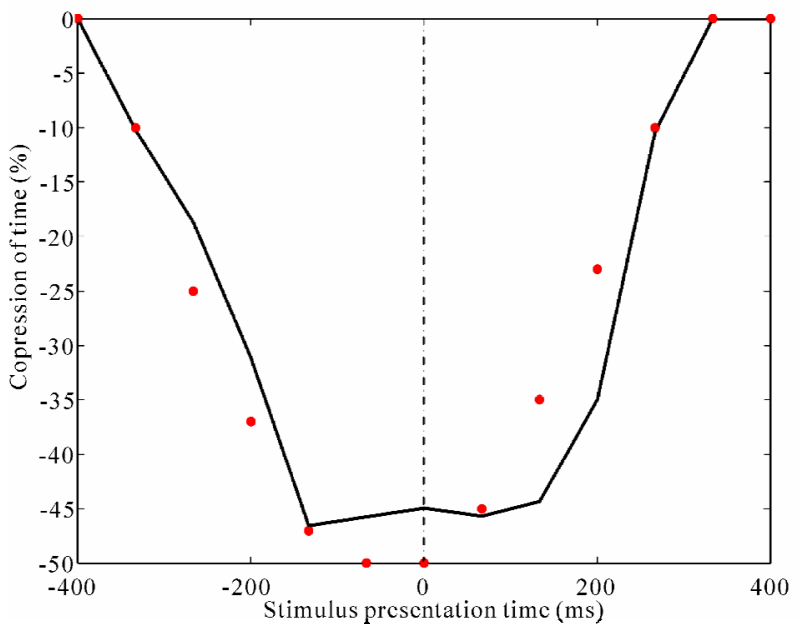

Figure 6. A cross-section of Figure 5 is shown for $\Delta T_{\mathrm{s}}=100$ $\mathrm{ms}$. The vertical dashed line indicates the time of the saccadic onset and the values on the $\mathrm{x}$ axis indicates the time delay of the second stimulus administration. In the $\mathrm{Y}$ axis the percentage of compression is reported. Red dots indicate the experimental observations and the continuous line represents the model estimation.

ters which ruled the synaptic dynamics. Finally a fundamental mechanism for the process under scrutiny has been identified as the interplay between the phase dynamics which characterize the neurons' activity and the synaptic dynamics. This model highlights how psychophysical and neurophysiological recent findings can drive interesting explanations for time and space perception at the saccadic time and for the visual perception in general. The simple implementation we propose for the data analysis can be exploited in two main directions. The former is the prediction of "new" and "unknown" 
visual effects during saccade, by the numerical simulation of arbitrary extreme setting conditions. Subsequent experimental inspections would drive to important refinements for the model and to new theoretical questions about the neural alghoritms used by the brain. The latter is represented by a clinical exploitation of the model, considering the evidences of the saccadic relate visual behaviors in neuropathological conditions as a reference dataset to be fitted $[23,29]$. We stress here that the modeling of time perception at perisaccadic events is a thriving field of research. It has two immediate and important benefits: the improved understanding of the processes that shape saccadic events and our improved ability to combine psychophysics and neuroscience to address research on clocks in the brain. Multiple Sclerosis, Hungtington's disease and Parkinson illness are only three of the multitude of clinical pathologies which affect both the neural phase dynamics and the synapses' behavior $[30,31,32]$. Describing the different pathologies in terms of their peculiar set of pathological effects allows us to easily map them into the model. This last crucial step would make of it a useful tool both to investigate the expected time and space visual perception impairment in those contexts and to explore new neural-related hypothesis for their explanation.

\section{ACKNOWLEDGEMENTS}

This work is financed by the EU 6 Framework Programme Project: Measuring and Modeling Relativistic-Like Effects in Brain and NCSs'. We thank David Burr, ConcettaMorrone, Marco Cicchini and Paola Binda of Pisa Vision Lab for suggestions.

\section{REFERENCES}

[1] Binda, P., Cicchini, G.M., Burr, D.C., and Morrone, M.C. (2009) Spatiotemporal distortions of visual perception at the time of saccades. Journal of Neuroscience, 29, 13147-13157.

[2] Burr, D. and Morrone, C. (2006) Perception: Transient disruptions to neural space time. Current Biology, 16, 848.

[3] Ibbotson, M., Crowder, N. and Price, N. (2005) Neural basis of time changes during saccades. Current Biology, 16, 834-836.

[4] Morrone, C., Ross, J. and Burr, D. (2005) Saccades cause compression of time as well as space. Nature Neuroscience, 8, 950-954.

[5] Ross, J., Morrone, C., Goldberg, E. and Burr, D. (2001) Changes in visualperception at the time of saccades. TRENDS in Neurosciences, 16, 472-479.

[6] Ross, J., Burr, D. and Morrone, C. (1996) Suppression of the magnocellular pathway during saccades. Behavioural Brain Research, 80, 1-8.

[7] Abeles, M. (1982) Local cortical circuits: An electrophysiological study. Springer, Berlin.

[8] Johnston, A., Arnold, D.H. and Nishida, S. (2006) Spatially localized distortions of event time. Current Biology,
16, 472-479.

[9] Rocca, E.D. and Burr, D.C. (2007) Large contrast effects in the discrimination of short temporal intervals. Perception 36 ECVP.

[10] Burr, D. and Morrone, C. (2006) Time perception: Space time in the brain. Current Biology, 16, 171-173.

[11] Lewis, P.A. and Walsh, V. (2005) Time perception: components of the brain's clock. Current Biology, 15, 389-391.

[12] Meck, W.H. (2005) Neuropsychology of timing and time perception. Brain Cognition, 58, 1-8.

[13] Ullner, E., Vicente, R., Pipa, G. and Garca-Ojalvo, J. (2008) Contour integration and synchronization in neuronal networks of the visual cortex. ICANN, Lecture Notes in Computer Science, 5164, 703-712.

[14] Orban, G.A., Van Essen, D. and Vanduffel, W. (2004) Comparative mapping of higher visual areas in monkeys and humans. Trends in Cognitive Sciences, 8, 315-324.

[15] Karmarkar, U. and Buonomano, D. (2007) Timing in the absence of clocks: Encoding time in neural network states. Neuron, 53, 427-438.

[16] Buonomano, D.V. (2000) Decoding temporal information: A model based on short-term synaptic plasticity. The Journal of Neuroscience, 20, 1129-1141.

[17] Gross, J., Schmitz. F., Schnitzler, I., Kessler, K., Shapiro, K., Hommel, B. and Schnitzler, A. (2004) Modulation of long-range neural synchrony reflects temporal limitations of visual attention in humans. Proceedings of the $\mathrm{Na}$ tional Academy Sciences, 101, 13050-13055.

[18] Gregoriou, G.G., Gotts, S.J., Zhou, H. and Desimone, R. (2009) High-frequency, long-range coupling between prefrontal and visual cortex during attention. Science, 324, 1207-1210.

[19] Mark, J. and Morrow, M.D. (1996) Craniotopic defects of smooth pursuit and saccadic eye movement. Neurology, 46, 514-521.

[20] Galletti, C., Battaglini, P. and Fattori, P. (1993) Parietal neurons encoding spatial locations in craniotopic coordinates. Experimental Brain Research, 96, 221-229.

[21] Graham, B.P. and Stricker, C. (2008) Short term plasticity provides temporal filtering at chemical synapses ICANN, Lecture Notes in Computer Science, 5164, 268-276.

[22] Hebb, D.O. (1949) The organization of behavior. Wily, New York.

[23] Btzel, K., Rottach, K. and Bttner, U. (1993) Normal and pathological saccadic dysmetria. Brain, 116, 337-353.

[24] Abarbanel, H., Gibb, L., Huerta, R., and Rabinovich, M. (2003) Biophysical model of synaptic plasticity dynamics. Biological Cybernetics, 89, 214-226.

[25] Dittman, J.S., Kreitzer, A.C. and Regehr, W.G. (2007) Interplay between facilitation, depression, and residual calcium at three presynaptic terminals, Journal of $\mathrm{Neu}$ roscience, 20, 1374-1385.

[26] Ford, J.M., Brian, J.R. and Mathalon, D.H. (2010) Assessing corollary discharge in humans using noninvasive neuron physiological methods. Nature Protocols, 5, 1160 -1168 .

[27] Kohonen, T. (1982) Self-organized formation of topologically correct feature maps. Biological Cybernetics, 43, 59-69.

[28] Guazzini, A., Lio, P., Conti, M. and Passarella, A. (2009) 
Information processing and timing mechanisms in vision. ICANN, Lecture Notes in Computer Science, 1, 325-334.

[29] McGivern, R., Gibson, J., Jwnnings, D., Lavery, K. and Montgomery,C. (2006) Normal and abnormal slowing of saccades: are they one and the same phenomenon? Annals of the New York Academy of Sciences, Neurobiology of eye movements: from molecules to behaviour, 956, 421-425.

[30] Huygena, P., Verhagenb, W., Hommesc, O. and Nicolasena, M. (1990) Short vestibule-ocular reflex time constants associated with oculomotor pathology in multiple sclerosis. Acta Oto-Laryngologica, 109, 25-33.

[31] Leigh, R. J., Parhad, I.M., Clark, A.W., Buettner-Ennever, J.A. and Folstein, S.E. (1985) Brainstem findings in Huntington's disease: Possible mechanisms for slow vertical saccades. Journal of the Neurological Sciences, 71, 247-256.

[32] Mosimann, U.P., Müri, R.M., Burn, D.J., Felblinger, J., O'Brien, J.T. and McKeith, I.G. (2005) Saccadic eye movement changes in Parkinson's disease dementia and dementia with Lewy bodies. Brain, 128, 1267-1276. 